\title{
Nickel acetylacetonate $\left[\mathrm{Ni}(\mathrm{acac})_{2}\right]$ and montmorillonite K-10 promoted regioselective $\mathrm{C}$-acylation of $\boldsymbol{\beta}$-enamino compounds
}

\author{
Hassan Sheibani* and Mohammad Seifi \\ Department of Chemistry, Shahid Bahonar University of Kerman, Kerman 76169, Iran \\ E-mail: hsheibani@mail.uk.ac.ir
}

\begin{abstract}
Regioselective acylation of enaminones in the presence of catalysts such as nickel acetylacetonate $\left[\mathrm{Ni}(\mathrm{acac})_{2}\right]$ and montmorillonite $\mathrm{K}-10$ to give $\mathrm{C}$-allylated products is reported. Nickel acetylacetonate $\left[\mathrm{Ni}(\mathrm{acac})_{2}\right]$ complexes of $\beta$-enamino compounds react with electrophiles as phenyl isocyanate and isothiocyanate at methin carbon, in good to excellent yields.
\end{abstract}

Keywords: Nickel acetylacetonate $\left[\mathrm{Ni}(\mathrm{acac})_{2}\right]$, montmorillonite $\mathrm{K}-10, \beta$-enamino compounds, phenyl isocyanate and phenyl isothiocyanate

\section{Introduction}

$\beta$-Enamino compounds are important compounds due to their application as 1,3-bielectrophilic and binucleophilic synthons in synthetic organic chemistry. ${ }^{1}$ These enaminones are versatile synthetic intermediates that combine the ambident nucleophilicity of enamines with the ambident electrophilicity of enones and have been extensively used for the preparation of a variety of heterocyclic systems. ${ }^{2,3}$ There are many reports on functionalization of enaminone in the literature by introduction of different substituents on the nitrogen, the $\alpha$-carbon and the $\beta$ carbonylic carbon atoms. These derivatives have been extensively used for the preparation of a variety of heterocyclic systems including some natural products and analogues. ${ }^{4,5}$

Regioselective C-functionalization of $\beta$-enamino compounds represents a considerable problem in organic synthesis. Singh et al. have recently reported regioselective allylation of enaminones using $\mathrm{CuCl}_{2}$ as catalyst to give $\mathrm{C}$-allylated products. ${ }^{6}$ It has been reported that reactions of $\beta$-enamino compounds with phenyl isocyanates and phenyl isothiocyanates in homogeneous media yield mixtures of $\mathrm{N}$ - and $\mathrm{C}$ - adducts. ${ }^{7}$ The difference of reactivity of the nucleophilic centers $(\mathrm{N}-$ and $\mathrm{C}-\alpha)$ and the absence of steric effects on the nitrogen in the starting material probably influenced in the regiochemistry of this reaction. In continuing our previous work on the reaction of $\beta$-enamino compounds with electrophiles, ${ }^{8,9}$ we now report a facile 
synthesis of 2-acyl-3-alkylamino-2-butenamide or 2-acyl-3-alkylamino-2-butenthioamide derivatives via regioselective acylation of $\beta$-enaminones using montmorillonite $\mathrm{K}-10$, or nickel acetylacetonate $\left[\mathrm{Ni}(\mathrm{acac})_{2}\right]$ as the catalyst. The catalytic properties of commercial aluminosilicates (zeolites and clays) suggested that a dual catalysis could be directly exerted by K10-montmorillonite, without incorporating any additional reactive species. ${ }^{10}$

Nickel acetyl acetonate is also known as bis(acetylacetonato)nickel (II). It has been used as a catalyst for oligomerization, reduction, cross-coupling, oxidation, conjugate addition, addition to multiple bonds and rearrangement reactions. It is commercially available. Alternatively, it can be prepared from potassium acetylacetonate and nickel (II) chloride. ${ }^{11}$

\section{Results and Discussion}

In order to study the reactivity of the $\beta$-enamino compounds with electrophiles, we used phenyl isocyanate or phenyl isothiocyanate as a electrophile with a series of selected acyclic $\beta$-enamino compounds (1a-f). Phenyl isocyanate and phenyl isothiocyanate permit the investigation of the competition at the nucleophilic centers, the $\alpha$-carbonylic carbon and the nitrogen of the $\beta$ enamino compounds in the presence of montmorillonite $\mathrm{K}-10$, or nickel acetylacetonate $\left[\mathrm{Ni}(\mathrm{acac})_{2}\right]$ as the catalyst. We have found that the metal acts as a 'protecting group' toward the nitrogen, so that, the nitrogen of the $\beta$-enamino compounds appears in general rather unreactive. This fact represents a distinct advantage in terms of chemoselectivity, because, electrophiles attack almost exclusively the methine carbon instead of the nitrogen (Scheme 1).

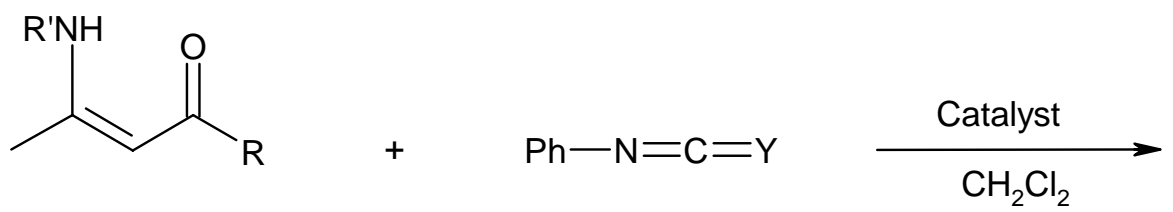

1a-f

1a: $\mathrm{R}=\mathrm{CH}_{3}, \mathrm{R}^{\prime}=\mathrm{H}$

1b: $\mathrm{R}=\mathrm{CH}_{3}, \mathrm{R}^{\prime}=\mathrm{C}_{6} \mathrm{H}_{5}$

1c: $\mathrm{R}=\mathrm{CH}_{3}, \mathrm{R}^{\prime}=4-\mathrm{MeC}_{6} \mathrm{H}_{4}$

$1 \mathrm{~d}: \mathrm{R}=\mathrm{CH}_{3}, \mathrm{R}^{\prime}=2,3-(\mathrm{Me})_{2} \mathrm{C}_{6} \mathrm{H}_{3}$

1e: $\mathrm{R}=\mathrm{C}_{6} \mathrm{H}_{5}, \mathrm{R}^{\prime}=\mathrm{n}-\mathrm{Butyl}$

1f: $\mathrm{R}=\mathrm{CH}_{3} \mathrm{CH}_{2} \mathrm{O}, \mathrm{R}^{\prime}=\mathrm{H}$
$2 \mathbf{a}, \mathbf{b}$

2a: $Y=O$

2b: $Y=S$<smiles>[R]C(=O)C(C([Y])=Nc1ccccc1)=C(C)N</smiles>

3 a-k

\section{Scheme 1}


Attempts to bring 1a and $\mathbf{1 b}$ to react with phenyl isocyanate or phenyl isothiocyanate to prepare either $\mathrm{C}$ or $\mathrm{N}$-allylated products of $\beta$-enamino compounds without using any catalyst in various solvents were mostly unsuccessful. The regiochemistry of these reactions depends on the $\mathrm{N}$-amino substituent. The reaction with the $\mathrm{NH}_{2}$ group leads preferentially to $\mathrm{N}$-acylated products, whereas reaction with the NHR-group (alkyl) yields C-acylated compounds preferentially. ${ }^{7}$ The acylation of $\beta$-enamino compounds $\mathbf{1 a - f}$ with phenyl isocyanate or phenyl isothiocyanate on montmorillonite K-10 gave selectively the C-acylated products 3a-k (Table1) after long reaction times and low yields were produced, while in the presence of nickel acetylacetonate $\left[\mathrm{Ni}(\mathrm{acac})_{2}\right]$ as a catalyst these products were obtained in short reaction times and excellent yields.

Table 1. Comparison of the catalytic efficiency of montmorillonite K-10 and nickel acetylacetonate $\left[\mathrm{Ni}(\mathrm{acac})_{2}\right]$ during regioselective $\mathrm{C}$-acylation of $\beta$-enamino compounds

\begin{tabular}{|c|c|c|c|c|c|c|c|}
\hline \multirow[t]{2}{*}{$\begin{array}{l}\text { Compd } \\
\text { No. }\end{array}$} & \multirow[t]{2}{*}{$\mathrm{R}$} & \multirow[t]{2}{*}{$\mathrm{R}^{\prime}$} & \multirow[t]{2}{*}{$\mathrm{Y}$} & \multicolumn{2}{|c|}{$\begin{array}{l}\text { Montmorillonite } \\
\text { K-10 }\end{array}$} & \multicolumn{2}{|c|}{$\begin{array}{c}\text { Nickel acetylacetonate } \\
{\left[\mathrm{Ni}(\mathrm{acac})_{2}\right]}\end{array}$} \\
\hline & & & & Yield (\%) & Time (h) & Yield (\%) & Time (h) \\
\hline $3 a$ & $\mathrm{CH}_{3}$ & $\mathrm{H}$ & $\mathrm{O}$ & 55 & 15 & 90 & 3 \\
\hline $3 b$ & $\mathrm{CH}_{3}$ & $\mathrm{H}$ & $\mathrm{S}$ & 62 & 15 & 93 & 3 \\
\hline $3 c$ & $\mathrm{CH}_{3}$ & $\mathrm{C}_{6} \mathrm{H}_{5}$ & $\mathrm{O}$ & 43 & 15 & 90 & 3 \\
\hline $3 d$ & $\mathrm{CH}_{3}$ & $\mathrm{C}_{6} \mathrm{H}_{5}$ & $\mathrm{~S}$ & 49 & 15 & 92 & 3 \\
\hline $3 e$ & $\mathrm{CH}_{3}$ & 4- $\mathrm{MeC}_{6} \mathrm{H}_{4}$ & $\mathrm{O}$ & 52 & 15 & 90 & 3 \\
\hline $3 f$ & $\mathrm{CH}_{3}$ & $4-\mathrm{MeC}_{6} \mathrm{H}_{4}$ & $\mathrm{~S}$ & 55 & 15 & 91 & 3 \\
\hline $3 g$ & $\mathrm{CH}_{3}$ & $2,3-(\mathrm{Me})_{2} \mathrm{C}_{6} \mathrm{H}_{3}$ & $\mathrm{~S}$ & 50 & 15 & 92 & 3 \\
\hline $3 h$ & $\mathrm{C}_{6} \mathrm{H}_{5}$ & n-Butyl & $\mathrm{O}$ & 63 & 12 & 92 & 2.5 \\
\hline $3 \mathrm{i}$ & $\mathrm{C}_{6} \mathrm{H}_{5}$ & n-Butyl & $\mathrm{S}$ & 65 & 12 & 95 & 2.5 \\
\hline $3 \mathrm{j}$ & $\mathrm{CH}_{3} \mathrm{CH}_{2} \mathrm{O}$ & $\mathrm{H}$ & $\mathrm{O}$ & 48 & 18 & 90 & 4 \\
\hline $3 \mathrm{k}$ & $\mathrm{CH}_{3} \mathrm{CH}_{2} \mathrm{O}$ & $\mathrm{H}$ & $\mathrm{S}$ & 53 & 18 & 92 & 4 \\
\hline
\end{tabular}

Montmorillonites are crystalline aluminosilicates, whose multilayered structure is characterized by the presence of Lewis acidic sites (localized mainly on the edges of the layer) ${ }^{12}$ and capable of protecting Lewis basic sites of $\beta$-enaminone (nitrogen and oxygen) (Scheme 2). 


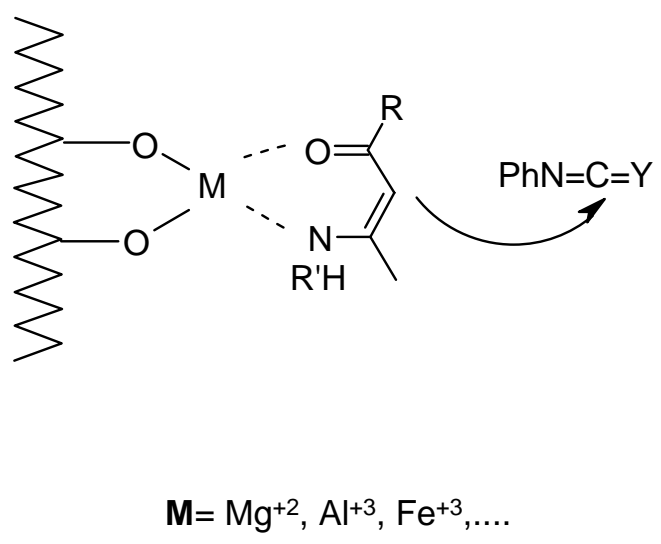

\section{Scheme 2}

Nickel acetylacetonate is even more effective at catalysing the carbon-carbon bond formation between $\beta$-enamino compounds with phenyl isocyanate and phenyl isothiocyanate. Coordination to metal centers not only can stabilize otherwise unstable organic fragments, but also can deeply modify their reactivity. ${ }^{13,14}$ The mechanism of the present reaction (scheme 1) initially proceeds through the coordination of the nitrogen and oxygen of the $\mathrm{C}=\mathrm{O}$ group with nickel acetylacetonate or Lewis acidic sites on montmorillonite K-10. Then methine carbon of $\beta$ enamino compounds attack to electrophile. The Final products will be synthesis by hydrogen shift followed by removal of the catalyst (scheme 3 ).

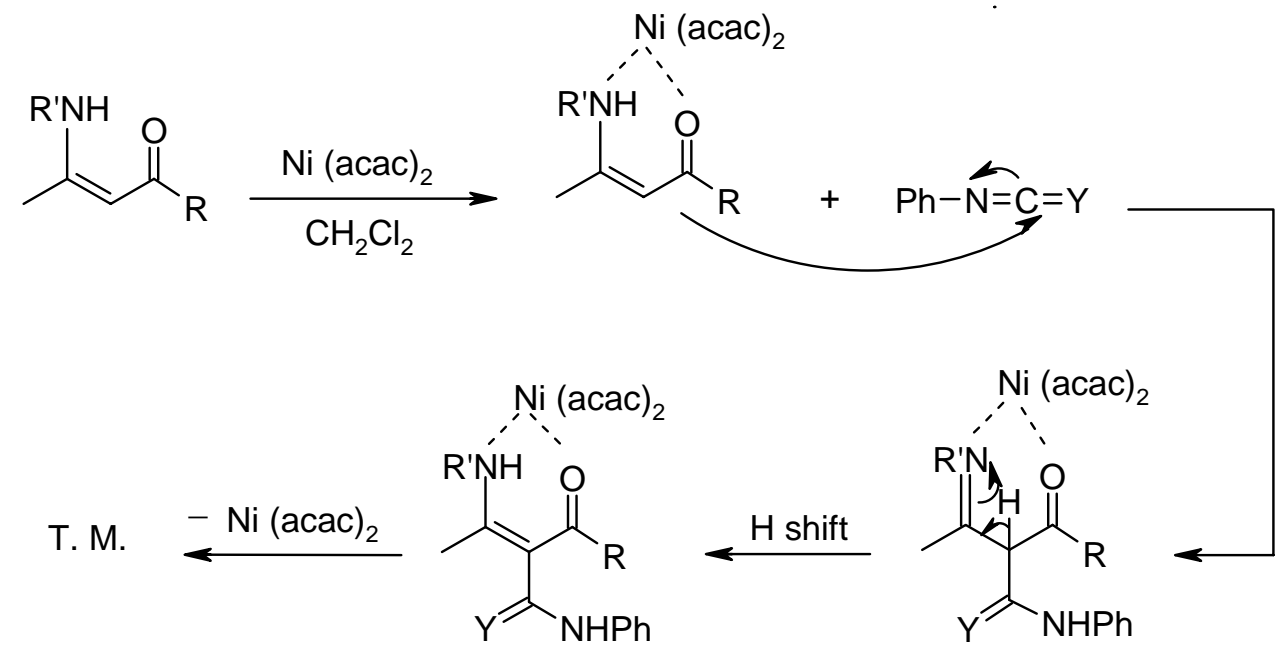

\section{Scheme 3}

These reactions were confirmed by ${ }^{1} \mathrm{H}$ NMR spectra as the signal for the olefinic proton at the $\alpha$ carbon atom of the $\beta$-enamino compounds disappear. In this study, all the products were characterized by melting point, IR, ${ }^{1} \mathrm{H}$ and ${ }^{13} \mathrm{C}$ NMR spectral data, as well as by their elemental 
analysis. The results obtained demonstrate that $\mathrm{Ni}(\mathrm{acac})_{2}$ is a good catalyst that allows the formation of a new carbon-carbon bond between $\beta$-enamino compounds with phenyl isocyanate and phenyl isothiocyanate. Thus, the presence of a metal centre can modify the normal reactivity exhibited by $\beta$-enamino compounds. The advantage of the procedure reported here are: high purity of products, high selectivity and easy workup.

\section{Experimental Section}

General Procedures. Melting points were measured on a Gallenkamp melting point apparatus and are uncorrected. IR spectra were measured on a Mattson 1000 FT-IR spectrometer. The proton and carbon NMR spectra were recorded with a BRUKER DRX-500 AVANCE spectrometer at 500 and $125.77 \mathrm{MHz}$, respectively. Mass spectra were recorded on a MSQP2000A Shimadzu mass spectrometer operating at an ionization potential of $70 \mathrm{eV}$. Elemental analyses were performed using a Heracus CHN-O-Rapid analyzer.

\section{General procedure for the preparation of $(E)$-2-acetyl-3-alkylamino- $N^{1}$-phenyl-2- buten- amide or butenethioamide derivatives (3a-k)}

Method A. Phenyl isocyanate or phenyl isothiocyanate $(2.5 \mathrm{mmol})$ was added dropwise to the $\beta$ enamino compounds $(2 \mathrm{mmol})$ dispersed on montmorillonite $\mathrm{K}-10(0.5 \mathrm{~g})$ and the mixture was stirred at $40{ }^{\circ} \mathrm{C}$ for the time reported in Table 1 (the progress of the reaction being monitored by TLC and using $n$-hexane/ethyl acetate as an eluent). The products were extracted by washing the montmorillonite with $\mathrm{CH}_{2} \mathrm{Cl}_{2}(5 \times 10 \mathrm{~mL})$, dried $\left(\mathrm{MgSO}_{4}\right)$, filtered and the solvent was removed in vacuo to yield the crude products which were purified by recrystallization from $96 \%$ ethanol.

Method B. A mixture of the $\beta$-enamino compounds $(2 \mathrm{mmol})$, phenyl isocyanate or phenyl isothiocyanate $(2 \mathrm{mmol})$ and $\mathrm{Ni}(\mathrm{acac})_{2}(0.03 \mathrm{~g})$ in $\mathrm{CH}_{2} \mathrm{Cl}_{2}(5 \mathrm{~mL})$ was refluxed with stirring. (the progress of the reaction being monitored by TLC and hexane/ethyl acetate was used as an eluent). After completion of the reaction the catalyst was separated from the reaction mixture by centrifugation. The excess $\mathrm{CH}_{2} \mathrm{Cl}_{2}$ was removed by evaporation and then the crude product was filtered, dried and recrystallized from $96 \%$ ethanol).

(E)-2-Acetyl-3-amino- $\boldsymbol{N}^{\mathbf{1}}$-phenyl-2-butenamide (3a). Pale yellow crystals. mp $153-155^{\circ} \mathrm{C}$. IR $\left(\mathrm{KBr}, v_{\max } \mathrm{cm}^{-1}\right): 3185,3135,3110\left(\mathrm{NH}_{2}, \mathrm{NH}\right), 1605(\mathrm{C}=\mathrm{O}) .{ }^{1} \mathrm{H}$ NMR $\left(500 \mathrm{MHz}, \mathrm{DMSO}-\mathrm{d}_{6}\right)$ : 13.15 (s, 1H, NH); 10.98 (s, 2H, NH); 7.95-6.85 (m, 5H, Ar); 2.19 (s, 3H, CH $)$; 2.02 (s, 3H, $\left.\mathrm{CH}_{3}\right) .{ }^{13} \mathrm{C}$ NMR (125 MHz, DMSO-d 6$) 192.21(\mathrm{C}=\mathrm{O}), 168.55,162.43\left(\mathrm{C}_{3}\right), 139.55,128.63$, 123.31, 119.36, $109.08\left(\mathrm{C}_{2}\right), 27.75\left(\mathrm{CH}_{3}\right), 20.01\left(\mathrm{CH}_{3}\right) . \mathrm{MS}(\mathrm{m} / \mathrm{z}): 218(12)\left(\mathrm{M}^{+}\right), 204(100), 175$ (15), 126 (58), 91 (8), 77 (65), 65 (15), 51 (43). Anal. Calcd. For $\mathrm{C}_{12} \mathrm{H}_{14} \mathrm{~N}_{2} \mathrm{O}_{2}$ : C, 66.04; H, 6.47; N, $12.84 \%$. Found : C, 65.79; H. 6.25; N;12.49.

(E)-2-Acetyl-3-amino- $\boldsymbol{N}^{\mathbf{1}}$-phenyl-2-butenethioamide (3b). Yellow crystals. mp $148-150^{\circ} \mathrm{C}$. IR $\left(\mathrm{KBr}, v_{\max } \mathrm{cm}^{-1}\right): 3180,3155,3120\left(\mathrm{NH}_{2}, \mathrm{NH}\right), 1595(\mathrm{C}=\mathrm{O}) .{ }^{1} \mathrm{H}$ NMR $\left(500 \mathrm{MHz}, \mathrm{DMSO}-\mathrm{d}_{6}\right)$ : $12.65(\mathrm{~s}, 1 \mathrm{H}) ; 11.18(\mathrm{~s}, 2 \mathrm{H}) ; 7.98-7.22(\mathrm{~m}, 5 \mathrm{H}, \mathrm{Ar}) ; 2.18\left(\mathrm{~s}, 3 \mathrm{H}, \mathrm{CH}_{3}\right) ; 2.04\left(\mathrm{~s}, 3 \mathrm{H}, \mathrm{CH}_{3}\right)$. 
${ }^{13} \mathrm{C}$ NMR (125 MHz, DMSO-d $\left.\mathrm{d}_{6}\right)$ 199.91(C=S), 190.80(C=O), 159.74( $\left.\mathrm{C}_{3}\right), 139.90,128.43$, 125.99, 122.97, 116.36( $\left.\mathrm{C}_{2}\right), 27.25\left(\mathrm{CH}_{3}\right), 19.75\left(\mathrm{CH}_{3}\right) . \mathrm{MS}(\mathrm{m} / \mathrm{z}): 234(15)\left(\mathrm{M}^{+}\right), 220(70), 191$ (15), 142 (43), 91 (9), 77 (75), 65 (23), 51 (43). Anal. Calcd. For $\mathrm{C}_{12} \mathrm{H}_{14} \mathrm{~N}_{2} \mathrm{OS}$ : C, 61.51; H, 6.02; N, $11.96 \%$. Found : C, 61.19; H. 5.90; N;11.59.

(E)-2-Acetyl-3-anilino- $N^{1}$-phenyl-2-butenamide (3c). Yellow crystals. mp $115-117^{\circ} \mathrm{C}$. IR $\left(\mathrm{KBr}, v_{\max } \mathrm{cm}^{-1}\right): 3250,3110(\mathrm{NH}), 1640,1589(\mathrm{C}=\mathrm{O}) .{ }^{1} \mathrm{H}$ NMR $\left(500 \mathrm{MHz}, \mathrm{DMSO}-\mathrm{d}_{6}\right): 13.29$ (s, 1H, NH); $10.30(\mathrm{~s}, 1 \mathrm{H}, \mathrm{NH}-\mathrm{C}=\mathrm{O}) ; 8.64-7.07$ (m, 10H, $\mathrm{Ar}) ; 2.13\left(\mathrm{~s}, 3 \mathrm{H}, \mathrm{CH}_{3}\right) ; 2.06(\mathrm{~s}, 3 \mathrm{H}$, $\left.\mathrm{CH}_{3}\right) .{ }^{13} \mathrm{C}$ NMR (125 MHz, DMSO-d $\left.\mathrm{d}_{6}\right) 193.80(\mathrm{C}=\mathrm{O}), 168.49(\mathrm{~N}-\mathrm{C}=\mathrm{O}), 160.80\left(\mathrm{C}_{3}\right), 140.35$, 138.46, 130.28, 129.62, 127.04, 125.84, 124.26, 120.08, 119.06, $111.07\left(\mathrm{C}_{2}\right), 28.49\left(\mathrm{CH}_{3}\right), 18.06$ $\left(\mathrm{CH}_{3}\right)$. MS (m/z): $294(7)\left(\mathrm{M}^{+}\right), 212(10), 202(75), 184(35), 160$ (60), $132(14), 118(39), 90$ (100), 77 (50), 65 (24), 51 (19). Anal. Calcd. For $\mathrm{C}_{18} \mathrm{H}_{18} \mathrm{~N}_{2} \mathrm{O}_{2}$ : C, 73.45; H, 6.16; N, $9.52 \%$. Found : C, 73.16; H. 6.02; N; $9.25 \%$.

(E)-2-Acetyl-3-anilino- $N^{1}$-phenyl-2-butenethioamide (3d). Yellow crystals. mp $116-118^{\circ} \mathrm{C}$. IR $\left(\mathrm{KBr}, v_{\max } \mathrm{cm}^{-1}\right): 3180,3110(\mathrm{NH}), 1589(\mathrm{C}=\mathrm{O}) .{ }^{1} \mathrm{H}$ NMR $\left(500 \mathrm{MHz}, \mathrm{DMSO}-\mathrm{d}_{6}\right): 13.29(\mathrm{~s}, 1 \mathrm{H}$, $\mathrm{NH}) ; 11.98$ (s, 1H, NH-C=S); 7.94-7.23 (m, 10H, Ar); 2.21 (s, 3H, $\left.\mathrm{CH}_{3}\right) ; 2.12$ (s, 3H, $\left.\mathrm{CH}_{3}\right)$. ${ }^{13} \mathrm{C}$ NMR (125 MHz, DMSO-d $\left.\mathrm{d}_{6}\right) 199.61(\mathrm{C}=\mathrm{S}), 192.89(\mathrm{C}=\mathrm{O}), 158.31\left(\mathrm{C}_{3}\right), 140.61,138.63$, 130.31, 129.46, 127.17, 126.81, 125.65, 123.77, $119.08\left(\mathrm{C}_{2}\right), 28.25\left(\mathrm{CH}_{3}\right), 17.81\left(\mathrm{CH}_{3}\right) . \mathrm{MS}$ (m/z): $310(10)\left(\mathrm{M}^{+}\right), 277(22), 175$ (58), 160 (95), 135 (100), 132 (34), 118 (23), 91 (8), 77 (75), 65 (15), 51 (43).(60), 263 (65), 188 (90), 160 (25), 138 (20), 104 (75), 77 (80), 51 (35). Anal. Calcd. For $\mathrm{C}_{18} \mathrm{H}_{18} \mathrm{~N}_{2} \mathrm{OS}$ : C, 69.65; H, 5.84; N, $9.02 \%$. Found : C, 69.86; H. 5.72; N; $9.15 \%$.

(E)-2-Acetyl- $\mathrm{N}^{1}$-phenyl-3-(4-toluidino)-2-butenamide (3e). White crystals. mp 146-148 ${ }^{\circ} \mathrm{C}$. IR $\left(\mathrm{KBr}, v_{\max } \mathrm{cm}^{-1}\right): 3250,3110(\mathrm{NH}), 1640,1589(\mathrm{C}=\mathrm{O}) .{ }^{1} \mathrm{H}$ NMR $\left(500 \mathrm{MHz}, \mathrm{DMSO}-\mathrm{d}_{6}\right): 13.23$ $(\mathrm{s}, 1 \mathrm{H}, \mathrm{NH}) ; 10.27(\mathrm{~s}, 1 \mathrm{H}, \mathrm{NH}-\mathrm{C}=\mathrm{O}) ; 8.64-6.97(\mathrm{~m}, 9 \mathrm{H}, \mathrm{Ar}) ; 2.32\left(\mathrm{~s}, 3 \mathrm{H}, \mathrm{CH}_{3}\right) ; 2.12(\mathrm{~s}, 3 \mathrm{H}$, $\left.\mathrm{CH}_{3}\right) ; 2.03\left(\mathrm{~s}, 3 \mathrm{H}, \mathrm{CH}_{3}\right) .{ }^{13} \mathrm{C}$ NMR $\left(125 \mathrm{MHz}, \mathrm{DMSO}_{-} \mathrm{d}_{6}\right) 193.52(\mathrm{C}=\mathrm{O}), 168.56(\mathrm{~N}-\mathrm{C}=\mathrm{O})$, $161.09\left(\mathrm{C}_{3}\right), 140.55,140.37,136.53,135.82,130.73,129.53,125.80,124.23,122.66,120.05$, 119.04, $111.42\left(\mathrm{C}_{2}\right), 28.45\left(\mathrm{CH}_{3}\right), 21.34\left(\mathrm{CH}_{3}\right), 18.02\left(\mathrm{CH}_{3}\right) . \mathrm{MS}(\mathrm{m} / \mathrm{z}): 308(10)\left(\mathrm{M}^{+}\right), 216$ (100), 198 (60), 189 (10), 174 (40), 158 (7), 144 (10), 132 (27), 119 (10), 106 (7), 93 (55), 77 (15), 65 (28), 51 (8). Anal. Calcd. For $\mathrm{C}_{19} \mathrm{H}_{20} \mathrm{~N}_{2} \mathrm{O}_{2}$ : C, 74.00; H, 6.54; N, 9.08 \%. Found : C, 73.86; H. 6.42; N; $8.75 \%$.

(E)-2-Acetyl- $N^{1}$-phenyl-3-(4-toluidino)-2-butenethioamide (3f). Green crystals. $\mathrm{mp} 93^{\circ} \mathrm{C}$. IR $\left(\mathrm{KBr}, v_{\max } \mathrm{cm}^{-1}\right): 3250,3110(\mathrm{NH}), 1640,1589(\mathrm{C}=\mathrm{O}) .{ }^{1} \mathrm{H}$ NMR (500 MHz, DMSO-d $\left.{ }_{6}\right): 13.24$ (s, 1H, NH); 11.98 (s, 1H, NH-C=S); 7.92-7.12 (m, 9H, Ar); 2.32 (s, 3H, CH $) ; 2.19$ (s, 3H, $\left.\mathrm{CH}_{3}\right), 2.08\left(\mathrm{~s}, 3 \mathrm{H}, \mathrm{CH}_{3}\right) .{ }^{13} \mathrm{C}$ NMR $\left(125 \mathrm{MHz}, \mathrm{DMSO}-\mathrm{d}_{6}\right) 199.73(\mathrm{C}=\mathrm{S}), 192.59(\mathrm{C}=\mathrm{O}), 158.54$ $\left(\mathrm{C}_{3}\right), 140.62,136.26,135.99,13.76,129.44,127.13,125.63,123.74,118.87\left(\mathrm{C}_{2}\right), 28.20\left(\mathrm{CH}_{3}\right)$, $21.35\left(\mathrm{CH}_{3}\right), 17.77\left(\mathrm{CH}_{3}\right) . \mathrm{MS}(\mathrm{m} / \mathrm{z}): 324(10)\left(\mathrm{M}^{+}\right), 291$ (15), 189 (30), 174 (58), $146(24), 135$ (100), 91 (39), 77 (87), 65 (28), 51 (58). Anal. Calcd. For $\mathrm{C}_{19} \mathrm{H}_{20} \mathrm{~N}_{2} \mathrm{OS}: \mathrm{C}, 70.34 ; \mathrm{H}, 6.21$; N, $8.63 \%$. Found : C, 70.13 ; H. 6.08 ; N; $8.27 \%$.

(E)-2-Acetyl-3-(2,3-dimethylanilino)- $N^{1}$-phenyl-2-butenethioamide (3g). Brown crystals. mp 82-83 ${ }^{\circ} \mathrm{C}$. IR ( $\left.\mathrm{KBr}, v_{\max } \mathrm{cm}^{-1}\right): 3350,3200(\mathrm{NH}), 1589(\mathrm{C}=\mathrm{O}) .{ }^{1} \mathrm{H}$ NMR (500 MHz, DMSO-d $\left.\mathrm{d}_{6}\right)$ : 13.11 (s, 1H, NH); 10.32 (s, 1H, NH-C=S); 7.14-6.97 (m, 8H, Ar); 2.27 (s, 3H, $\left.\mathrm{CH}_{3}\right) ; 2.12$ (s, 
$\left.3 \mathrm{H}, \mathrm{CH}_{3}\right) ; 2.07$ (s, 3H, $\left.\mathrm{CH}_{3}\right) ; 1.81\left(\mathrm{~s}, 3 \mathrm{H}, \mathrm{CH}_{3}\right) ;{ }^{13} \mathrm{C}$ NMR (125 MHz, DMSO-d $\left.\mathrm{d}_{6}\right) 200.86(\mathrm{C}=\mathrm{O})$, $192.66(\mathrm{C}=\mathrm{S}), 159.76\left(\mathrm{C}_{3}\right), 138.81,137.27,132.86,129.07,126.76,125.05,116.79\left(\mathrm{C}_{2}\right), 27.99$ $\left(\mathrm{CH}_{3}\right), 20.91\left(\mathrm{CH}_{3}\right), 17.54\left(\mathrm{CH}_{3}\right), 14.76\left(\mathrm{CH}_{3}\right) . \mathrm{MS}(\mathrm{m} / \mathrm{z}): 338(5)\left(\mathrm{M}^{+}\right), 305(8), 243(15), 146$ (12), 136 (10), 121 (15), 105 (10), 95 (16), 81 (48), 69 (100), 55 (30). Anal. Calcd. For $\mathrm{C}_{20} \mathrm{H}_{22} \mathrm{~N}_{2} \mathrm{OS}$ : C, 70.97; H, 6.55; N, 8.28 \%. Found : C, 70.68; H. 6.39; N; $7.95 \%$.

(E)-2-Benzoyl-3-(butylamino)- $\boldsymbol{N}^{\mathbf{1}}$-phenyl-2-butenamide (3h). Yellow crystals. mp $165-166^{\circ} \mathrm{C}$. IR $\left(\mathrm{KBr}, v_{\max } \mathrm{cm}^{-1}\right): 3220,3150(\mathrm{NH}), 1589(\mathrm{C}=\mathrm{O}) .{ }^{1} \mathrm{H}$ NMR (500 MHz, DMSO-d $\left.{ }_{6}\right): 12.20(\mathrm{~s}$, $1 \mathrm{H}, \mathrm{NH}) ; 9.95(\mathrm{~s}, 1 \mathrm{H}, \mathrm{NH}-\mathrm{C}=\mathrm{O}) ; 8.65(\mathrm{~s}, 1 \mathrm{H}) ; 7.52-6.95(\mathrm{~m}, 10 \mathrm{H}, \mathrm{Ar}) ; 3.41\left(\mathrm{t}, 2 \mathrm{H},{ }^{3} J_{\mathrm{H}-\mathrm{H}}=5.4\right.$, $\left.\mathrm{CH}_{2}\right) ; 2.14$ (s, $\left.3 \mathrm{H}, \mathrm{CH}_{3}\right) ; 1.60$ (m, $\left.2 \mathrm{H}, \mathrm{CH}_{2}\right) ; 1.43\left(\mathrm{~m}, 2 \mathrm{H}, \mathrm{CH}_{2}\right) ; 0.95\left(\mathrm{t}, 3 \mathrm{H},{ }^{3} J_{\mathrm{H}-\mathrm{H}}=0.6, \mathrm{CH}_{3}\right)$; ${ }^{13} \mathrm{C}$ NMR (125 MHz, DMSO-d $\left.\mathrm{d}_{6}\right) 189.87(\mathrm{C}=\mathrm{O}), 168.96(\mathrm{~N}-\mathrm{C}=\mathrm{O}), 167.12\left(\mathrm{C}_{3}\right), 143.32,140.32$, 129.64, 129.21, 128.50, 127.64, 123.80, 120.10, $119.04\left(\mathrm{C}_{2}\right), 43.30,32.06,20.40,17.20,14.42$. MS (m/z): $336(8)\left(\mathrm{M}^{+}\right), 244$ (100), 212 (9), 129 (10), $119(5), 105$ (75), 93 (68), 77 (45), 65 (14). Anal. Calcd. For $\mathrm{C}_{21} \mathrm{H}_{24} \mathrm{~N}_{2} \mathrm{O}_{2}$ : C, 74.97; H, 7.19; N, $8.33 \%$. Found : C, 74.76; H. 7.02; N; $8.15 \%$.

(E)-2-Benzoyl-3-(butylamino)- $N^{1}$-phenyl-2-butenethioamide (3i). Yellow crystals. mp 133$135^{\circ} \mathrm{C}$. IR $\left(\mathrm{KBr}, v_{\max } \mathrm{cm}^{-1}\right)$ : 3220, $3150(\mathrm{NH}), 1589(\mathrm{C}=\mathrm{O}) .{ }^{1} \mathrm{H}$ NMR $\left(500 \mathrm{MHz}\right.$, DMSO-d $\left.\mathrm{d}_{6}\right)$ : $12.35(\mathrm{~s}, 1 \mathrm{H}, \mathrm{NH}) ; 11.60(\mathrm{~s}, 1 \mathrm{H}, \mathrm{NH}-\mathrm{C}=\mathrm{S}) ; 7.55-7.15(\mathrm{~m}, 10 \mathrm{H}, \mathrm{Ar}) ; 3.44\left(\mathrm{t}, 2 \mathrm{H},{ }^{3} \mathrm{~J}_{\mathrm{H}-\mathrm{H}}=5.1, \mathrm{CH}_{2}\right)$; $2.25\left(\mathrm{~s}, 3 \mathrm{H}, \mathrm{CH}_{3}\right) ; 1.61\left(\mathrm{~m}, 2 \mathrm{H}, \mathrm{CH}_{2}\right) ; 1.44\left(\mathrm{~m}, 2 \mathrm{H}, \mathrm{CH}_{2}\right) ; 0.96\left(\mathrm{t}, 3 \mathrm{H},{ }^{3} J_{\mathrm{H}-\mathrm{H}}=0.6, \mathrm{CH}_{3}\right) .{ }^{13} \mathrm{C} \mathrm{NMR}$ $\left(125 \mathrm{MHz}, \mathrm{DMSO}-\mathrm{d}_{6}\right) 199.40(\mathrm{C}=\mathrm{S}), 188.71(\mathrm{C}=\mathrm{O}), 165.29\left(\mathrm{C}_{3}\right), 143.52,140.58,129.60$, 129.14, 128.22, 127.33, 126.75, 123.93, $116.59\left(\mathrm{C}_{2}\right), 43.44,32.07,20.44,17.38,14.45$. MS (m/z): $352(8)\left(\mathrm{M}^{+}\right), 319$ (15), 217 (40), 200 (25), 188 (20), 174 (19), 160 (15), 135 (70), 105 (57), 91 (35), 77 (100), 68 (18), 51 (33). Anal. Calcd. For $\mathrm{C}_{21} \mathrm{H}_{24} \mathrm{~N}_{2} \mathrm{OS}$ : C, 71.56; H, 6.86; N, $7.95 \%$. Found : C, 71.39 ; H. 6.67; N; $7.69 \%$.

Ethyl(Z)-3-amino-2-(anilinocarbonyl)-butenoate (3j). Pale yellow crystals. mp $120-122^{\circ} \mathrm{C}$. IR $\left(\mathrm{KBr}, v_{\max } \mathrm{cm}^{-1}\right): 3198,3125,3110\left(\mathrm{NH}_{2}, \mathrm{NH}\right), 1625(\mathrm{C}=\mathrm{O}) .{ }^{1} \mathrm{H}$ NMR (500 MHz, DMSO-d 6 ): 12.15 (s, $1 \mathrm{H}, \mathrm{NH}) ; 10.58\left(\mathrm{~s}, 2 \mathrm{H}, \mathrm{NH}_{2}\right) ; 7.55-6.75$ (m, 5H, Ar); 4.07 (q, 2H, J= 7.3, $\left.\mathrm{CH}_{2}\right), 2.08$ (s, $\left.3 \mathrm{H}, \mathrm{CH}_{3}\right) ; 1.15\left(\mathrm{t}, 3 \mathrm{H}, J=7.3, \mathrm{CH}_{3}\right) \cdot{ }^{13} \mathrm{C}$ NMR $\left(125 \mathrm{MHz}, \mathrm{DMSO}-\mathrm{d}_{6}\right) 169.04(\mathrm{C}=\mathrm{O}), 167.50$, $151.19\left(\mathrm{C}_{3}\right), 139.71,128.48,122.56,119.46,118.04\left(\mathrm{C}_{2}\right), 59.15,24.21,14,22 . \mathrm{MS}(\mathrm{m} / \mathrm{z}): 248(7)$ $\left(\mathrm{M}^{+}\right)$, 203(34), 156(100), 91 (11), 77 (55), 65 (18), 51 (41). Anal. Calcd. For $\mathrm{C}_{13} \mathrm{H}_{16} \mathrm{~N}_{2} \mathrm{O}_{3}: \mathrm{C}$, $62.89 ; \mathrm{H}, 6.50 ; \mathrm{N}, 11.28 \%$. Found : C, 62.59; H. 6.36; N;11.01.

Ethyl (E)-3-amino-2-(anilinocarbothioyl)-butenoate (3k). Yellow crystals. mp $136-137^{\circ} \mathrm{C}$. IR $\left(\mathrm{KBr}, v_{\max } \mathrm{cm}^{-1}\right): 3170,3140,3115\left(\mathrm{NH}_{2}, \mathrm{NH}\right), 1615(\mathrm{C}=\mathrm{O}) .{ }^{1} \mathrm{H}$ NMR $\left(500 \mathrm{MHz}, \mathrm{DMSO}-\mathrm{d}_{6}\right)$ : 12.05 (s, $1 \mathrm{H}) ; 11.46(\mathrm{~s}, 2 \mathrm{H}) ; 7.82-7.27$ (m, 5H, $\mathrm{Ar}) ; 4.01$ (q, $\left.2 \mathrm{H}, J=7.2, \mathrm{CH}_{2}\right), 2.01\left(\mathrm{~s}, 3 \mathrm{H}, \mathrm{CH}_{3}\right)$; $1.12\left(\mathrm{t}, 3 \mathrm{H}, J=7.2, \mathrm{CH}_{3}\right) \cdot{ }^{13} \mathrm{C}$ NMR (125 MHz, DMSO-d 6$)$ 198.00(C=S), 166.45(C=O), 159.16( $\left.\mathrm{C}_{3}\right), 140.19,128.33,125.62,123.12,103.44\left(\mathrm{C}_{2}\right), 58.43,19.99,14.42 . \mathrm{MS}(\mathrm{m} / \mathrm{z}): 264(12)$ $\left(\mathrm{M}^{+}\right), 219(32), 191$ (14), 172(23), 91 (12), 77 (55), 65 (27), 51 (43). Anal. Calcd. For $\mathrm{C}_{13} \mathrm{H}_{16} \mathrm{~N}_{2} \mathrm{O}_{2} \mathrm{~S}: \mathrm{C}, 59.07 ; \mathrm{H}, 6.10 ; \mathrm{N}, 10.60 \%$. Found : C, 58.72; H. 5.96; N;10.29.

\section{Acknowledgements}


The authors express appreciation to the Shahid Bahonar University of Kerman Faculty Research Committee for its support of this investigation

\section{References}

1. Rappoport, Z. The Chemistry of Enamines, John Wiley: New York, 1994 (Part 1).

2. Elasser, A. A; El-Khair, A. A. Tetrahedron 2003, 59, 8463.

3. Kascheres, C. J. Braz. Chem. Soc. 2003, 14, 945.

4. Lue, P.; Greenhill, J. V. Adv. Heterocycl. Chem. 1997, 67, 207.

5. Baraldi, P. G.; Barco, A.; Benetti, S.; Pollini, G. P.; Simoni, D. Synthesis 1987, 857.

6. Singh, S. J.; Singh, O. M. Tetrahedron Lett. 2008, 49, 3991.

7. Maquestiau, A.; Vanden Eynde, J. J.; Monclus, M. Bull. Soc. Chim. Belg. 1985, 94, 575.

8. Sheibani, H.; Islami, M. R.; Hosseininasab, F. A.; Hassanpour, A. Arkivoc 2006, (xv), 175.

9. Abaszadeh, M.; Sheibani, H.; Saidi, K. J. Heterocycl.Chem. 2009. 46, 96.

10. Soriente, A.; Arienzo, R.; Margherita De Rosa, M. D.; Palombi, L.; Spinella, A.; Scettri, A. Green Chem. 1999. 157.

11. Canoira, L.; Rodriguez, J. G. J. Heterocycl.Chem. 1985, 22, 1511.

12. Palombi, L.; Bonadies, F.; A. Scettri, A. J. Mol. Catal. 1999, 140, 47.

13. Suk-Ku Kang, S.-K.; Ryu, H.-C.; Lee, S-W. J. Chem. Soc., Perkin Trans. 1 1999, 2661.

14. Shi, Y.-C.; Cheng, H.-J.; Zhang, S.-H. Polyhedron 2008, 27, 3331. 